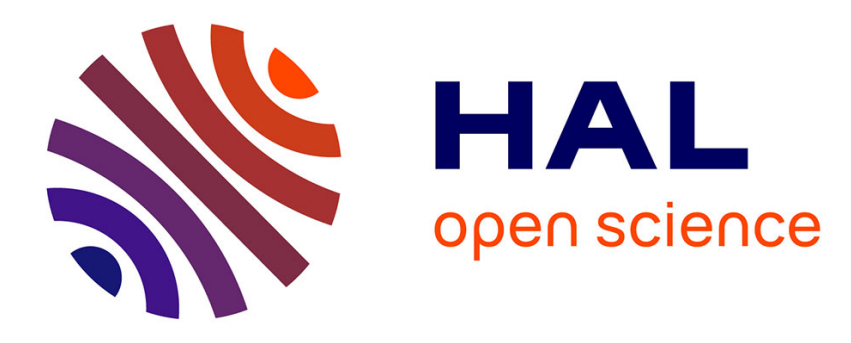

\title{
Analyse dynamique du déplacement de domaines magnétiques par des gradients de champ
}

H. Jouve, J.P. Jadot

\section{To cite this version:}

H. Jouve, J.P. Jadot. Analyse dynamique du déplacement de domaines magnétiques par des gradients de champ. Revue de Physique Appliquée, 1975, 10 (2), pp.51-59. 10.1051/rphysap:0197500100205100 . jpa-00243880

\section{HAL Id: jpa-00243880 https://hal.science/jpa-00243880}

Submitted on 1 Jan 1975

HAL is a multi-disciplinary open access archive for the deposit and dissemination of scientific research documents, whether they are published or not. The documents may come from teaching and research institutions in France or abroad, or from public or private research centers.
L'archive ouverte pluridisciplinaire HAL, est destinée au dépôt et à la diffusion de documents scientifiques de niveau recherche, publiés ou non, émanant des établissements d'enseignement et de recherche français ou étrangers, des laboratoires publics ou privés. 
Classification

Physics Abstracts

8.550

\section{ANALYSE DYNAMIQUE \\ DU DÉPLACEMENT DE DOMAINES MAGNÉTIQUES PAR DES GRADIENTS DE CHAMP}

\author{
H. JOUVE et J. P. JADOT \\ Laboratoire d'Electronique et de Technologie de l'Informatique \\ Centre d'Etudes Nucléaires, B. P. 85, 38041 Grenoble Cedex, France
}

(Reçu le 5 septembre 1974)

\begin{abstract}
Résumé. - On établit le système d'équations différentielles du second ordre qui gouverne le déplacement de domaines magnétiques cylindriques de section circulaire dans un gradient de champ magnétique de forme quelconque. On donne une vérification expérimentale des calculs dans le cas d'une bulle de $70 \mu$ de diamètre déplacée par un conducteur électrique. L'analyse des conditions de transfert d'une bulle par un conducteur montre que le déplacement par courants bipolaires est impossible. On trouve que le temps de transfert est inversement proportionnel au courant de commande et que la largeur optimum d'un conducteur est de une à deux fois le diamètre des bulles. D'autres effets plus faibles sont mis en évidence, tels que l'action du champ de polarisation sur le temps de transfert et la position d'équilibre et l'influence du courant de commande sur le rayon et la position d'équilibre.

Abstract. - The system of differential equations which governs the motion of circular cylindrical magnetic domains is established and we present an experimental verification of the calculation in the case of a bubble $70 \mu$ in diameter moved by an electric conductor. Quantitative analysis of bubble transfer by a conductor shows that motion by bipolar currents is not possible. The transfer time is found to be inversely proportional to the current amplitude, and optimum width for the conductor is shown to be between one and two bubble diameter. We also report other, smaller effects such as the action of the polarizing field on the transfer time and the equilibrium position and the influence of the current amplitude on the radius and equilibrium position.
\end{abstract}

1. Introduction. - Depuis plusieurs années, un gros effort scientifique et technologique a été consacré à l'étude des bulles magnétiques pour leur utilisation comme éléments mémoires.

Le déplacement des bulles est obtenu par la génération locale de gradients de champ magnétique à l'aide de conducteurs électriques et de motifs de permalloy. A l'heure actuelle, les dessins et l'optimisation des circuits sont réalisés par essais successifs en raison de l'absence d'une analyse quantitative complète des phénomènes.

En raison de la complexité des calculs, les efforts ont porté jusqu'à présent, soit sur l'analyse statique des bulles avec leur environnement, soit sur l'introduction de modèles phénoménologiques pour l'étude dynamique.

Sous l'angle statique, certains auteurs ont étudié l'interaction bulle-permalloy [2], [3], puis ces calculs ont été raffinés en tenant compte de la variation de rayon de la bulle [4], et en prenant un modèle bidimensionnel de l'aimantation des barreaux de permalloy [5].

D'autres travaux ont été consacrés à l'étude de la force exercée par un conducteur sur des bulles [6], [7].
Abordant la propagation des bulles sous son aspect dynamique des auteurs ont utilisé des modèles phénoménologiques pour rendre compte des limitations en champ [8] ou en fréquence [9] des phénomènes de propagation.

Dans l'étude présentée ici, on n'étudiera que le cas de la propagation par conducteurs électriques en raison de sa plus grande simplicité. La méthode utilisée fait la synthèse des deux précédentes, en ce sens que le déplacement est traité au moyen d'équations différentielles dépendantes du temps; le champ magnétique est calculé à chaque instant en fonction de la position et du rayon de la bulle.

Le principe retenu a été de conduire analytiquement les calculs aussi loin que possible, puis de recourir au calcul numérique pour terminer les intégrations et résoudre les équations différentielles.

2. Etablissement des équations différentielles de propagation. - 2.1 CONDITIONS D'ÉQUILIBRE STATIQUE D'UNE BULlE. - La situation est décrite par la figure 1. On suppose que :

1) Le champ d'anisotropie $H_{\mathrm{K}}$ est perpendiculaire 


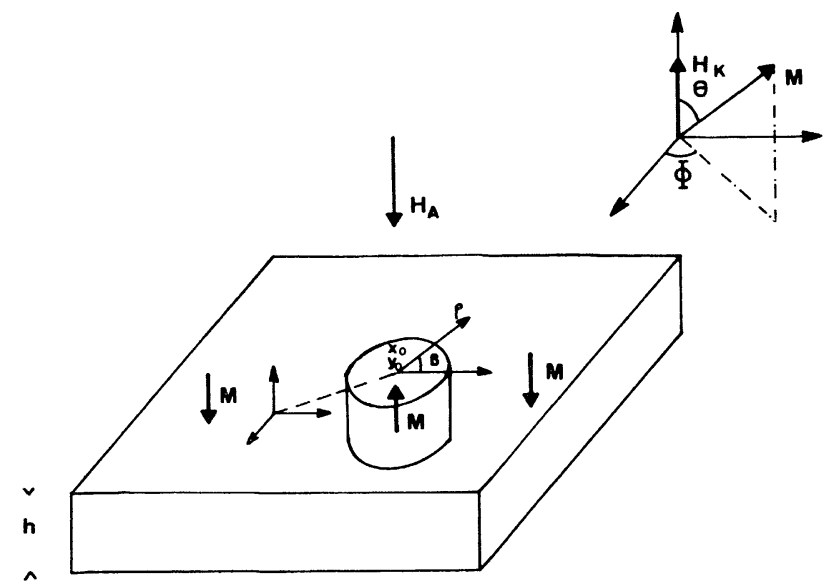

FIG. 1. - Domaine magnétique circulaire, centré en $x_{0}, y_{0}$ dans une plaquette supposée de surface infinie.

au plan de la lame, et son module est beaucoup plus grand que $4 \pi M, M$ étant la densité d'aimantation du matériau.

2) Le domaine magnétique est un cylindre d'axe // à $H_{\mathrm{K}}$, de section circulaire.

3) Une paroi de Bloch contribue à l'énergie totale par sa densité d'énergie par unité de surface $\sigma_{\mathrm{W}}$.

4) L'échantillon a une épaisseur $h$ uniforme, et sa surface est supposée infinie.

5) L'énergie totale peut s'écrire comme la somme des trois termes.

- L'énergie de la paroi de Bloch, $E_{\mathrm{W}}$ qui s'exprime à l'aide de :

$$
E_{\mathrm{w}}=\int_{S_{\mathrm{b}}} \sigma_{\mathrm{w}} \dot{d}_{\mathrm{S}}
$$

L'énergie $E_{\mathrm{A}}$ d'interaction avec le champ magnétique appliqué $H_{\mathrm{A}}$ :

$$
E_{\mathrm{A}}=-2 \int_{V_{\mathrm{b}}} \mathbf{M} \cdot \mathbf{H}_{\mathrm{A}} \mathrm{d} V_{\mathrm{b}} .
$$

L'énergie démagnétisante $E_{\mathrm{D}}$, dont on ne retient que la partie qui est fonction du rayon et des coordonnées de la bulle :

$$
E_{\mathrm{D}}=-\int_{V_{\mathrm{b}}} \mathbf{M} \cdot \mathbf{H}_{\mathrm{D}} \mathrm{d} V_{\mathrm{b}}
$$

$H_{\mathrm{D}}:$ champ démagnétisant.

$$
E_{\mathrm{T}}=E_{\mathrm{A}}+E_{\mathrm{W}}+E_{\mathrm{D}} .
$$

Les conditions d'équilibre statique d'une bulle sont obtenues en minimisant $E_{\mathrm{T}}$ par rapport à son rayon et aux coordonnées de son centre.

2.2 EQuAtions DiffÉRENTIELles GOUVERNANT LE DÉPLACEMENT D'UNE BULlE. - On utilise l'équation phénoménologique proposée par Gilbert [10] qui décrit le mouvement des spins dans un champ magnétique $\mathbf{H}$ :

$$
\frac{\mathrm{d} \mathbf{M}}{\mathrm{d} t}=\gamma \mathbf{M} \wedge\left(\mathbf{H}-\eta \frac{\mathrm{d} \mathbf{M}}{\mathrm{d} t}\right)
$$

$\gamma$ : facteur gyromagnétique,

$\eta$ : constante d'amortissement.

Cette équation peut s'obtenir à partir d'une fonction de Lagrange $L$ et d'une fonction de dissipation de Rayleigh $G$ dont la densité volumique est :

$$
g=\frac{1}{2} M \eta\left[\left(\frac{\mathrm{d} \theta}{\mathrm{d} t}\right)^{2}+\sin ^{2} \theta\left(\frac{\mathrm{d} \Phi}{\mathrm{d} t}\right)^{2}\right]
$$

$\theta$ et $\Phi$ sont les angles polaires du vecteur moment magnétique (Fig. 1).

Le lagrangien vaut: $L=T-U, T$ étant l'énergie cinétique et $U$ l'énergie potentielle.

On choisit de prendre pour $\tau$ (densité d'énergie cinétique par unité de volume) [11]:

$$
\tau=-\frac{M}{\gamma} \frac{\mathrm{d}(\cos \theta)}{\mathrm{d} t} \cdot \Phi .
$$

Les équations du mouvement s'obtiennent par les équations d'Euler correspondantes :

$$
\frac{\mathrm{d}}{\mathrm{d} t} \frac{\partial L}{\partial \dot{q}}-\frac{\partial L}{\partial q}=-\frac{\partial G}{\partial \dot{q}}
$$

$q$ étant une des variables indépendantes du mouvement.

Pour une bulle circulaire de centre $x_{0}$ et $y_{0}$ de rayon $R$, ces équations sont :

$$
\left.\begin{array}{l}
\frac{\mathrm{d}}{\mathrm{d} t} \frac{\partial T}{\partial \dot{x}_{0}}+\frac{\partial U}{\partial x_{0}}=-\frac{\partial G}{\partial \dot{x}_{0}} \\
\frac{\mathrm{d}}{\mathrm{d} t} \frac{\partial T}{\partial \dot{y}_{0}}+\frac{\partial U}{\partial y_{0}}=-\frac{\partial G}{\partial \dot{y}_{0}} \\
\frac{\mathrm{d}}{\mathrm{d} t} \frac{\partial T}{\partial R}+\frac{\partial U}{\partial R}=-\frac{\partial G}{\partial \dot{R}}
\end{array}\right\}
$$

L'énergie potentielle $U$ est l'énergie $E_{\mathrm{T}}$ de (1).

Avec les hypothèses faites sur la nature du domaine, $G$ est :

$$
\begin{aligned}
G=\frac{h}{2} & \cdot M \cdot \eta \int_{0}^{\infty} \int_{0}^{2 \pi} \times \\
& \times\left[\left(\frac{\mathrm{d} \theta(\rho, \beta)}{\mathrm{d} t}\right)^{2}+\sin ^{2} \theta(\rho, \beta)\left(\frac{\mathrm{d} \Phi}{\mathrm{d} t}\right)^{2}\right] \rho \mathrm{d} \rho \mathrm{d} \beta
\end{aligned}
$$

$\beta$ étant l'angle polaire et $\rho$ le rayon vecteur d'origine $x_{0}$, $y_{0}$ et de composantes $\left(x_{0}+R \cos \beta, y_{0}+R \sin \beta\right)$ (Fig. 1).

On prend pour la forme de la paroi une expression simple [11]:

$$
\sin \theta=\left[\operatorname{ch}\left(\frac{\rho-R}{\delta}\right)\right]^{-1}
$$

$\delta$ définissant l'épaisseur de la paroi. 
Après intégration, on obtient pour la fonction de dissipation :

$$
G=\frac{1}{2} b\left[\frac{1}{2}\left(\frac{\mathrm{d} x_{0}}{\mathrm{~d} t}\right)^{2}+\frac{1}{2}\left(\frac{\mathrm{d} y_{0}}{\mathrm{~d} t}\right)^{2}+\left(\frac{\mathrm{d} R}{\mathrm{~d} t}\right)^{2}\right]
$$

où :

$$
b=\frac{4 \pi M_{\mathrm{S}} h \cdot R}{\mu} ;
$$

$\mu=\delta / \eta$ étant la mobilité.

Pour l'énergie cinétique :

$$
T=\int_{0}^{2 \pi} \mathrm{d} \beta \int_{0}^{\infty} \rho \mathrm{d} \rho\left(-\frac{M}{\gamma}\right)\left(\frac{\mathrm{d} \cos \theta}{\mathrm{d} t}\right)^{2} \Phi
$$

soit :

$$
\begin{aligned}
T & =\frac{1}{2} m\left[\frac{1}{2}\left(\frac{\mathrm{d} x_{0}}{\mathrm{~d} t}\right)^{2}+\frac{1}{2}\left(\frac{\mathrm{d} y_{0}}{\mathrm{~d} t}\right)^{2}+\left(\frac{\mathrm{d} R}{\mathrm{~d} t}\right)^{2}\right] \\
m & =\frac{\sigma_{\mathrm{W}} \cdot R \cdot h}{\sqrt{2} A \gamma^{2}}
\end{aligned}
$$

$A$ est la constante d'échange.

Le système différentiel (2) devient :

$$
\left.\begin{array}{l}
\frac{m}{2} \ddot{x}_{0}+\frac{b}{2} \dot{x}_{0}=-\frac{\partial E_{\mathrm{A}}}{\partial x_{0}} \\
\frac{m}{2} \ddot{y}_{0}+\frac{b}{2} \dot{y}_{0}=-\frac{\partial E_{\mathrm{A}}}{\partial y_{0}} \\
m \ddot{R}+b \dot{R}=-\frac{\partial E_{\mathrm{A}}}{\partial R}-\frac{\partial E_{\mathrm{D}}}{\partial R}-\frac{\partial E_{\mathrm{W}}}{\partial R}
\end{array}\right\}
$$

La résolution de (3) donne les valeurs $x_{0}(t), y_{0}(t), R(t)$ qui décrivent le mouvement et la déformation de la bulle en fonction du temps.

2.3 VALEURS DES COEFFICIENTS DU SYSTÈME DIFFÉRENTIEL. - Deux paramètres physiques du matériau à bulles interviennent comme coefficients dans le système d'équations différentielles que nous avons établi; il s'agit de la masse équivalente de paroi $m$ et du coefficient d'amortissement $b$ lié directement à la mobilité $\mu$.

Bien que de nombreux travaux théoriques aient été consacrés à l'analyse de ces paramètres, en raison du point de vue adopté ici, on utilisera seulement des données expérimentales.

Pour comparer l'importance du terme d'origine inertielle lié à $m$, à celui d'origine dissipatif lié à $b$, supposons que la bulle soit animée d'un mouvement de pulsation harmonique, d'amplitude $r$, sans que sa position d'équilibre $\left(x_{0}, y_{0}\right)$ ne soit affectée.

Le système (3) devient alors :

$$
m \frac{\mathrm{d}^{2}(r \sin \omega t)}{\mathrm{d} t^{2}}+b \frac{\mathrm{d}(r \sin \omega t)}{t}=-\frac{\partial E_{\mathrm{T}}}{\partial R} .
$$

Le rapport entre la force d'origine inertielle $F_{\mathrm{i}}$ et celle d'origine dissipative $F_{\mathrm{D}}$ est :

$$
\frac{F_{\mathrm{i}}}{F_{\mathrm{D}}}=\frac{m \omega}{b} \text {. }
$$

Dans les orthoferrites, Rossol a montré [13] que ce rapport était très faible et que par conséquent on pouvait négliger dans l'équation différentielle les termes du deuxième ordre.

Pour les grenats de terres rares, plusieurs mesures de résonance des parois ont été faites [14], [15], [16] pour déterminer la valeur de $m$.

Ces différentes mesures donnent pour $m$ un ordre de grandeur de :

$$
m \simeq 10^{-10} \mathrm{~g} \mathrm{~cm}^{-2} .
$$

Avec :

$$
\begin{aligned}
\omega & =2 \pi \times 10^{5} \\
4 \pi M_{\mathrm{S}} & =200 \mathrm{G} \\
\mu & =10^{3} \mathrm{~cm} \cdot \mathrm{s}^{-1} \cdot \mathrm{Oe}^{-1} \\
\frac{F_{\mathrm{i}}}{F_{\mathrm{D}}} & =2 \times 10^{-3} .
\end{aligned}
$$

Nous négligerons donc dans le système (3) les termes du second ordre. Le mouvement des bulles ne dépend alors que du terme d'amortissement $b$, c'est-à-dire de la mobilité.

De nombreuses méthodes ont été mises au point pour déterminer la mobilité des parois dans les films ferrimagnétiques.

Dans certaines [13], on observe le déplacement d'une paroi plane soumise à un gradient de champ magnétique, variant périodiquement en fonction du temps avec une pulsation $\omega$. La fréquence au-delà de laquelle la paroi ne peut plus suivre la perturbation qui lui est imposée, donne la valeur de la mobilité du matériau.

Cette méthode présente l'inconvénient de nécessiter des hautes fréquences pour l'étude des grenats.

D'autres auteurs utilisent la réponse d'une paroi plane à une impulsion rectangulaire de champ magnétique [17], [18].

Une autre méthode d'utilisation très simple est celle qui consiste à faire disparaître les bulles sous l'action d'une impulsion de champ magnétique [19]. Son principal défaut est que la vélocité de la paroi est très fortement fonction du diamètre de la bulle, ce qui nécessite une analyse complexe pour obtenir la mobilité de la paroi.

Ces trois premières techniques présentent l'inconvénient que les mesures sont faites sur des parois qui ont des vitesses dépendant du temps et qu'en présence de non-linéarité, il est très difficile sinon impossible d'extraire la relation entre la vitesse et le champ.

Ceci peut être évité par la méthode [20] dans laquelle on observe par stroboscopie le mouvement d'une bulle autour d'un disque de permalloy. Cette situation se rapproche beaucoup de celle où une bulle se propage sur un circuit avec des motifs, mais l'évaluation exacte de la mobilité n'est pas possible car on ne sait pas calculer le gradient de champ exercé par le disque de permalloy. 
La technique du déplacement des bulles dans un gradient de champ [21], à rayon de bulle constant, est considérée actuellement comme la meilleure pour les grenats épitaxiés.

Ces différentes méthodes ont été utilisées pour la mesure des mobilités dans les orthoferrites et dans les grenats.

Au cours des expériences, une différence fondamentale est apparue entre ces deux types de matériaux : alors que les quatre premières méthodes donnent des résultats concordants pour les orthoferrites, une grande dispersion des résultats ainsi qu'un phénomène de non-linéarité ont été constatés dans les grenats [22], [23], [24]. Un modèle basé sur un processus de conversion dynamique des parois avec apparition et nucléation de lignes de Bloch pendant le déplacement a été proposé pour expliquer ces anomalies [25].

L'attribution d'une mobilité bien définie étant donc très difficile dans les grenats, les vérifications expérimentales et les calculs présentés ici seront faits avec des matériaux orthoferrites. Ils peuvent être étendus à d'autres classes de matériaux (grenats, magnétiques amorphes) pour lesquels la mobilité est suffisamment bien connue.

3. Action d'un conducteur électrique sur une bulle. 3.1 MISE EN FORME DES ÉQUATIONS ET CALCUL. Considérons un conducteur électrique de section rectangulaire (Fig. 2) d'épaisseur $d$ et de largeur $B$, situé

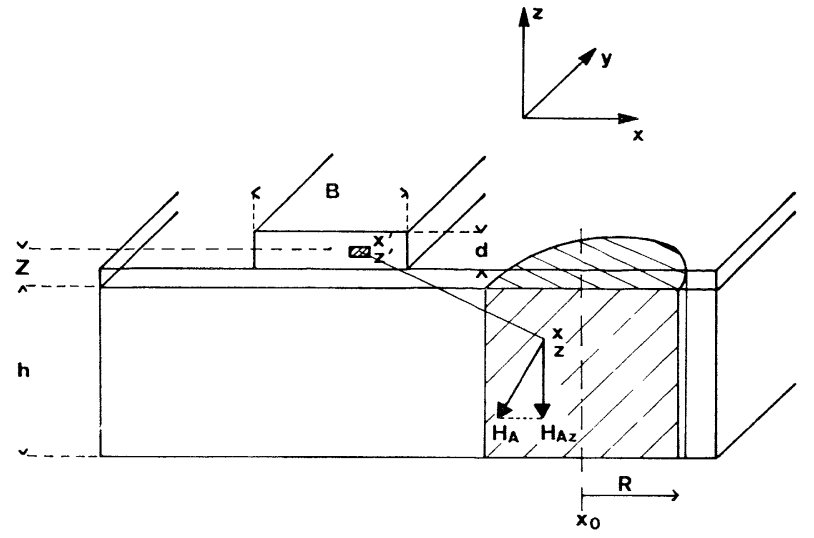

FIG. 2. - Champ $\mathbf{H}_{\mathrm{A}}$ créé par un élément infinitésimal d'un conducteur électrique au niveau du matériau dans lequel se déplace la bulle.

à une distance $Z$ de la partie supérieure du matériau à bulles. On suppose le conducteur infini dans la direction $y$. Le système (3) se réduit alors à :

$$
\left.\begin{array}{l}
\frac{b}{2} \dot{x}_{0}=-\frac{\partial E_{\mathrm{A}}}{\partial x_{0}} \\
b \dot{R}=-\frac{\partial E_{\mathrm{A}}}{\partial R}-\frac{\partial E_{\mathrm{D}}}{\partial R}-\frac{\partial E_{\mathrm{W}}}{\partial R}
\end{array}\right\}
$$

$E_{\mathrm{A}}$ est l'énergie d'interaction entre la bulle et le champ magnétique $H_{\mathrm{A}}$ créé par le conducteur, son expression formelle est :

$$
\begin{aligned}
& E_{\mathrm{A}}=-2 \int_{V_{\mathrm{b}}} \mathbf{H}_{\mathrm{A}} \cdot \mathbf{M} \mathrm{d} V_{\mathrm{b}} \\
& E_{\mathrm{A}}=-2 M \cdot h \int_{\sigma_{\mathrm{B}}}<H_{\mathrm{A} z}>\mathrm{d} x \mathrm{~d} y
\end{aligned}
$$

$\sigma_{\text {B }}$ section de la bulle :

$$
V_{\mathrm{B}}=\sigma_{\mathrm{B}} \times h
$$

et

$$
<H_{\mathrm{A} z}>=\frac{1}{h} \int_{-h}^{0} H_{\mathrm{A} z} \mathrm{~d} z .
$$

Puisque $\mathbf{M}$ est parallèle à l'axe $z$, seule importe la composante verticale de $\mathbf{H}_{\mathrm{A}}$ (Fig. 2). Le champ créé par un élément $\mathrm{d} x^{\prime} \mathrm{d} z^{\prime}$ de la section du conducteur au point $(x, z)$ vaut :

$$
\mathrm{d} H_{\mathrm{A} z}(x, z)=2 j \frac{x-x^{\prime}}{\left(x-x^{\prime}\right)^{2}+\left(z-z^{\prime}\right)^{2}} \mathrm{~d} x^{\prime} \mathrm{d} z^{\prime}
$$

$j$ étant la densité du courant :

$$
j=\frac{I}{B \cdot d} .
$$

Le champ total est :

$$
\left.<H_{\mathrm{A} z}\right\rangle=\frac{1}{h} \int_{-h}^{0} \mathrm{~d} z \int_{Z-\frac{d}{2}}^{Z+\frac{d}{2}} \int_{-\frac{B}{2}}^{+\frac{B}{2}} \mathrm{~d} H_{\mathrm{A} z} .
$$

En appelant

$$
\begin{array}{ll}
X_{-}=x-\frac{B}{2} & Z_{-}=Z-\frac{h}{2} \\
X_{+}=x+\frac{B}{2} & Z_{+}=Z+\frac{h}{2}
\end{array}
$$

l'expression de $\left\langle H_{\mathrm{Az}}\right\rangle$ est, en supposant $d \ll h$ :

$$
\begin{aligned}
& <H_{\mathrm{A} z}(x)>=\frac{I}{B h}\left[-Z_{-} \ln \left(\frac{X_{-}^{2}+Z_{-}^{2}}{X_{+}^{2}+Z_{-}^{2}}\right)+\right. \\
& +2 X_{-} \operatorname{Arctg}\left(-\frac{Z_{-}}{X_{-}}\right)-2 X_{+} \operatorname{Arctg}\left(-\frac{Z_{-}}{X_{+}}\right) \\
& +Z_{+} \ln \left(\frac{X_{-}^{2}+Z_{+}^{2}}{X_{+}^{2}+Z_{+}^{2}}\right)-Z X_{-} \operatorname{Arctg}\left(-\frac{Z_{+}}{X_{-}}\right) \\
& \left.+2 X_{+} \operatorname{Arctg}\left(-\frac{Z_{+}}{X_{+}}\right)\right] .
\end{aligned}
$$

L'énergie $E_{\mathrm{A}}$ est alors :

$$
E_{\mathrm{A}}=-2 \cdot M \cdot \int_{0}^{R} \rho \mathrm{d} \rho \int_{0}^{2 \pi} \mathrm{d} \beta<H_{\mathrm{Az}}>.
$$

Dans le nouveau système de coordonnées cylindriques :

$$
x=x_{0}+\rho \cos \beta \text {. }
$$


Les dérivées partielles de $E_{\mathrm{A}}$ par rapport à $x_{0}$ et $R$ sont dans le cas général :

$$
\begin{aligned}
\frac{\partial E_{\mathrm{A}}}{\partial R}=-2 . & M \cdot R \int_{0}^{2 \pi} \mathrm{d} \beta \times \\
\times & <H_{\mathrm{A} z}\left(x_{0}+R \cos \theta, y_{0}+R \sin \theta\right)>
\end{aligned}
$$

et :

$$
\begin{aligned}
\frac{\partial E_{\mathrm{A}}}{\partial x_{0}}=- & 2 M \int_{0}^{2 \pi} \mathrm{d} \beta \int_{0}^{R} \rho \frac{\partial}{\partial x_{0}} \times \\
& \times<H_{\mathrm{Az}}\left(x_{0}+\rho \cos \beta, y_{0}+\rho \sin \beta\right)>\mathrm{d} \rho .
\end{aligned}
$$

En remarquant que :

$$
\frac{\partial H_{\mathrm{A}}}{\partial x_{0}}=\frac{\partial H_{\mathrm{A}}}{\partial \rho} \times \frac{1}{\cos \beta}
$$

on peut dans la deuxième expression effectuer l'intégration par rapport à $\rho$, ce qui fait que les deux termes $\partial E_{\mathrm{A}} / \partial R$ et $\partial E_{\mathrm{A}} / \partial x_{0}$ ne contiendront plus qu'une intégration par rapport à $\beta$.

Cette dernière est réalisée numériquement par la méthode de Romberg. Ce calcul permet donc de déterminer à chaque instant la vitesse du centre de la bulle et de la variation du rayon.

Un programme de calcul numérique a été mis au point qui, à partir d'une position initiale $\left(x_{\mathrm{i}}, R_{\mathrm{i}}\right)$, détermine l'évolution en fonction du temps de $x_{0}$ et de $R$ et les valeurs d'équilibre à l'arrivée $x_{\mathrm{F}}$ et $R_{\mathrm{F}}$.

Les quantités qui intéressent le concepteur de circuit sont l'état initial $\left(x_{\mathrm{i}}, R_{\mathrm{i}}\right)$, l'état final $\left(x_{\mathrm{F}}, R_{\mathrm{F}}\right)$ et le temps $\tau$ nécessaire pour ce déplacement. Les paramètres sur lesquels on peut agir sont : $B$ la largeur du conducteur, $I$ l'intensité de l'impulsion et enfin il faut tenir compte de l'influence du champ de polarisation $H_{\mathrm{p}}$.

La situation est schématisée sur le tableau I.

\begin{tabular}{ccc} 
& TABLEAU I & \\
Valeurs initiales & $\begin{array}{c}\text { Valeurs } \\
\text { recherchées }\end{array}$ & $\begin{array}{c}\text { Paramètres } \\
\text { gouvernables }\end{array}$ \\
\hline$x_{\mathrm{i}}$ & - & - \\
$R_{\mathrm{i}}$ & $x_{\mathrm{F}}-x_{\mathrm{i}}$ & $B$ \\
& $R_{\mathrm{F}}$ & $I$ \\
$\tau$ & $H_{\mathrm{p}}$
\end{tabular}

Nous allons donner des résultats obtenus par le calcul numérique pour cerner l'influence des paramètres $B, I, H_{\mathrm{p}}$ sur les valeurs recherchées $x_{\mathrm{F}}-x_{\mathrm{i}}$, $R_{\mathrm{F}}$ et $\tau$.

\subsection{VÉRIFICATIONS EXPÉRIMENTALES DU PROGRAMME} DE CALCUL NUMÉRIQUE. - Les vérifications expérimentales sont faites par observation stroboscopique, ce qui permet de mesurer la position et la dimension de la bulle à tout instant. Les bulles sont suffisamment éloignées sur le circuit pour que leurs interactions soient négligeables; le mouvement est donc le résultat de l'action de l'élément de propagation sur la bulle.
Le circuit qui sert à la vérification du calcul (Fig. 3) utilise le principe de la génération à partir d'un domaine parent et de la propagation par des motifs de permalloy.

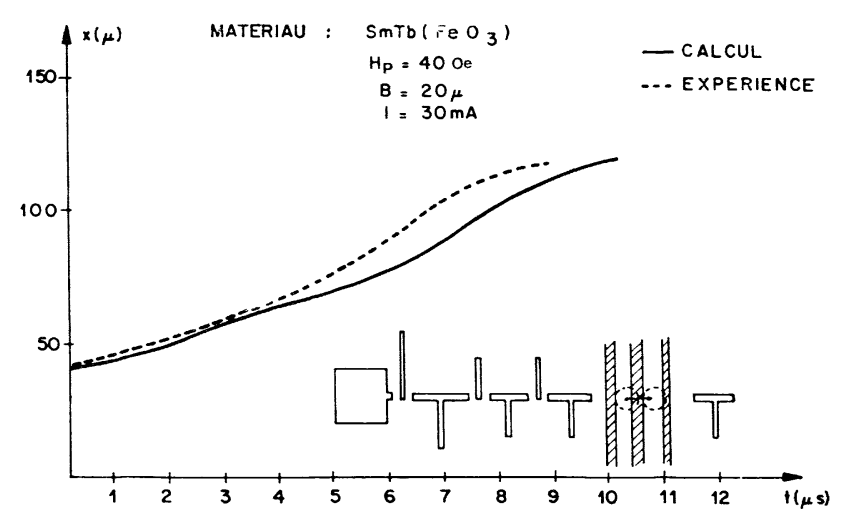

FIG. 3. - Vérification expérimentale de calcul, le diamètre de la bulle est de l'ordı de $70 \mu$.

Afin de pouvoir tester le déplacement par conducteurs seulement, il faut éviter la présence de motifs de permalloy ; aussi le circuit est-il interrompu et trois conducteurs électriques alimentés successivement assurent la propagation des bulles. Les mesures sont faites lors de la propagation par le troisième conducteur au cours de laquelle les motifs de permalloy sont les plus éloignés. La fréquence est de $12,5 \mathrm{kHz}$, la fenêtre laser a une durée de $2 \mu$ s, les observations et les mesures sont réalisées sur un écran de télévision. Sur la figure 3, on peut comparer une courbe expérimentale et la courbe calculée correspondante. L'allure du déplacement $x(t)$, ainsi que la position d'équilibre, sont parfaitement bien rendues par le calcul. Il n'y a pas cependant coïncidence exacte entre les deux, en raison des simplifications du modèle mathématique et des incertitudes expérimentales. La précision sur l'échelle des temps est limitée par l'électronique de commande des impulsions, qui, à $12 \mathrm{kHz}$, sont échelonnées toutes les $1,2 \mu \mathrm{s}$ et par la durée et la forme de la fenêtre laser.

En dépit de simplifications, il apparaît que le calcul numérique donne une représentation quantitative satisfaisante du comportement de bulles soumises à des gradients de champ magnétique.

L'utilisation systématique de ces calculs va permettre de déterminer l'influence des différents paramètres qui ont été définis plus haut.

3. 3 INFLUENCE DU POINT DE DÉPART $x_{\mathbf{i}}$. - L'examen de la courbe donnant le champ magnétique vertical créé par un conducteur et intégré sur le volume d'une bulle (Fig. 4), montre qu'il existe pour le domaine magnétique un point d'équilibre situé en $\mathrm{B}$ et que selon que la bulle arrive de la région du point $\mathrm{C}$ ou de la région du point $\mathrm{A}$, les modules des vitesses seront différents et les signes opposés.

Plus précisément, on va étudier comment la distance entre le point de départ de la bulle et le centre du conducteur va influencer le temps de transfert et la 


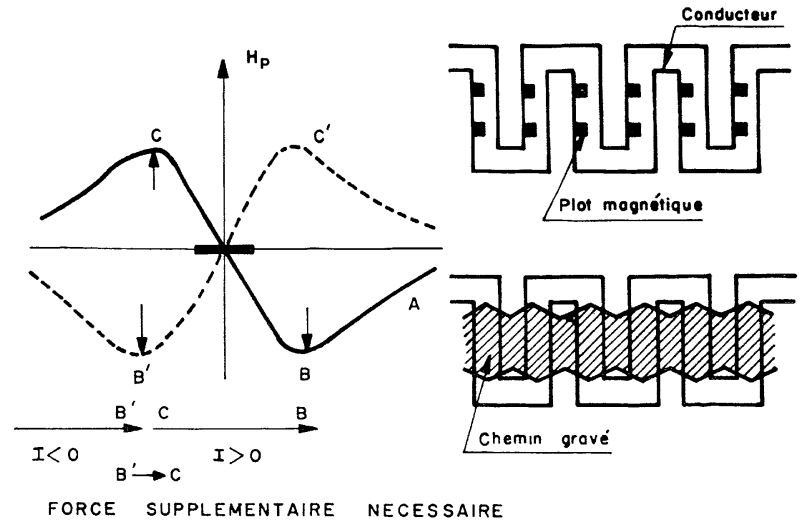

Fig. 4. - Champ moyen $H_{\mathrm{p}}$ créé par un conducteur intégré sur le volume de la bulle. Quand $I$ est négatif, le point d'équilibre est en $\mathrm{B}^{\prime}$, quand $I$ est positif il est en $\mathrm{B}$ et la bulle ne peut y être amené qu'à partir du point $\mathrm{C}$. Il faut exercer une force supplémentaire entre $\mathrm{B}^{\prime}$ et $\mathrm{C}$ à l'aide de plots magnétiques ou de chemins gravés.

vitesse moyenne de la bulle. Celle-ci est définie comme le quotient entre la distance parcourue $x_{\mathrm{F}}-x_{\mathrm{i}}$ et le temps de transfert $\tau$.

Si le conducteur était filiforme et la bulle ponctuelle, le champ magnétique serait de la forme :

$$
H=\frac{K}{x} .
$$

L'énergie d'interaction entre la bulle et le conducteur est alors :

$$
\begin{gathered}
E=\int M \cdot H \mathrm{~d} v=M \frac{K}{x} \\
\frac{\partial E}{\partial x}=-\frac{M K}{x^{2}} \\
\frac{b}{2} \frac{\mathrm{d} x}{\mathrm{~d} t}=\frac{M \cdot K}{x^{2}} \\
\tau=\int_{x_{\mathrm{i}}}^{x_{F}} \frac{b}{2} \frac{x^{2} \mathrm{~d} x}{M K}=\frac{b}{6} \frac{x_{\mathrm{F}}^{3}-x_{\mathrm{i}}^{3}}{M K}
\end{gathered}
$$

et la vitesse moyenne :

$$
\bar{v}=\frac{x_{\mathrm{F}}-x_{\mathrm{i}}}{\tau}=\frac{6 M K}{b\left(x_{\mathrm{F}}^{2}+x_{\mathrm{F}} x_{\mathrm{i}}+x_{\mathrm{i}}^{2}\right)} .
$$

Si $x_{\mathrm{i}} \gg x_{\mathrm{F}}$

$$
\bar{v}=\frac{6 M K}{b x_{i}^{2}} .
$$

On doit donc s'attendre à ce que loin du conducteur, la vitesse moyenne d'une bulle augmente comme l'inverse du carré de la distance parcourue.

Par contre, si le point de départ de la bulle est à proximité du conducteur, les effets du diamètre de la bulle et de la largeur du conducteur sont primordiaux. On a soumis une bulle de diamètre $70 \mu$ au champ créé par un conducteur de $70 \mu$ de large. On a étudié le point d'équilibre, le temps de transfert $\tau$ et la vitesse de déplacement. Les résultats sont sur la figure 5. On a porté en abscisse les valeurs des points de départ $x_{\mathrm{i}}$ et en ordonnées le temps de transfert et la vitesse moyenne correspondant à chacun de ces points de départ.

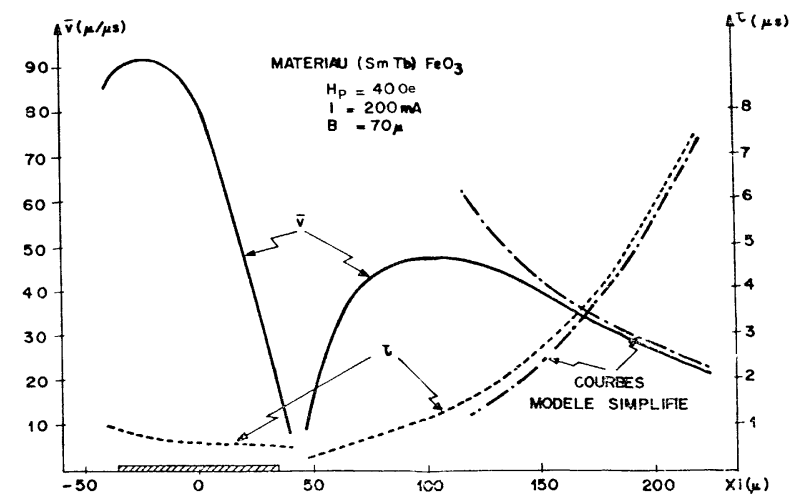

FIG. 5. - Vitesse moyenne $\bar{v}$ et temps de transfert $\tau$, pour différents points de départ $x_{\mathrm{i}}$. Le point d'équilibre final est en $x_{\mathrm{F}}=45 \mu$.

On remarque d'abord que loin du conducteur (pour $x_{\mathrm{i}}>150 \mu$ ) le comportement de la bulle obéit assez bien à l'analyse simple précédente, le temps de transfert $\tau \sim x_{\mathrm{i}}^{3}$ et la vitesse $\bar{v} \sim 1 / x^{2}$.

On note ensuite que pour parcourir des distances moyennes $\left|x_{\mathrm{F}}-x_{\mathrm{i}}\right| \simeq 30$ à $80 \mu$, il est beaucoup plus avantageux d'utiliser le conducteur en faisant passer la bulle par-dessous depuis les $x$ négatifs plutôt que de l'attirer depuis les $x$ positifs. De cette façon on peut atteindre des vitesses moyennes deux fois plus grandes.

Enfin à proximité du conducteur pour

$$
\left|x_{\mathrm{F}}-x_{\mathrm{i}}\right|<20 \mu,
$$

la vitesse moyenne chute très vite, ceci est dû à la forme très arrondie du puits de potentiel vers lequel la bulle est entraînée (Fig. 4).

\subsection{INFLUENCE DE LA LARGEUR DU CONDUCTEUR. --} En premier lieu la largeur du conducteur de transfert va agir sur le point d'équilibre final de la bulle. On a tracé les courbes de transfert pour des largeurs de conducteurs allant de $2 \mu$ à $400 \mu$, et on a porté l'abscisse et le rayon des points d'équilibre en fonction de la demilargeur des conducteurs (Fig. 6). Pour des conducteurs très étroits le point d'équilibre est déterminé par le rayon de la bulle, et pour des conducteurs très larges, le point d'équilibre est donné par la demi-largeur du conducteur, c'est-à-dire qu'il se trouve iuste au bord de celui-ci. Si on utilise le conducteur de la manière la plus efficace, c'est-à-dire en faisant passer la bulle d'un bord à l'autre, le chemin qui pourra être parcouru est donc directement proportionnel à la largeur des conducteurs.

Sur la même figure, on a représenté l'évolution de la vitesse moyenne d'une bulle partant du centre du conducteur en fonction de la largeur de ce conducteur, cette vitesse qui reste constante tant que le diamètre de 
MATERIAU (SmTb) $\mathrm{FeO}_{3}$

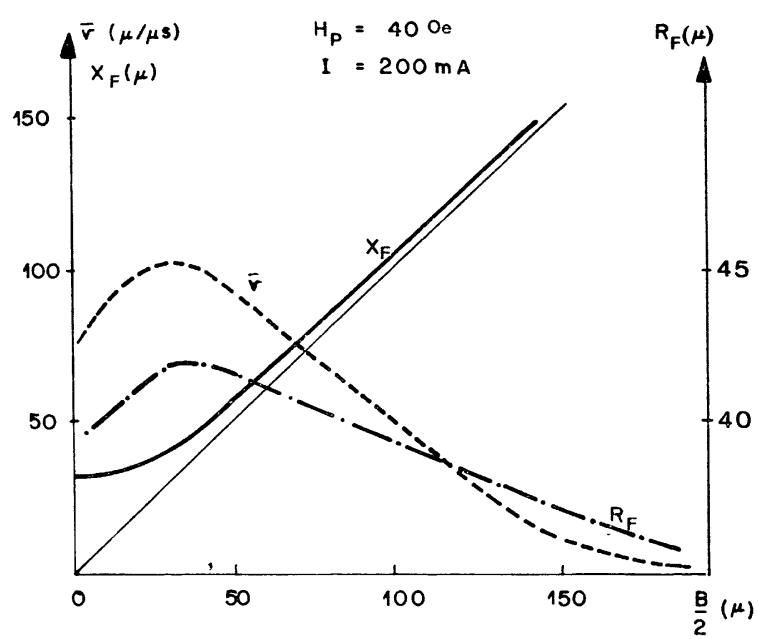

Fig. 6. - Influence de la largeur du conducteur $B$ sur la vitesse moyenne $\bar{v}$ et les coordonnées d'équilibre final $x_{\mathrm{F}}$ et $R_{\mathrm{F}}$.

la bulle est de l'ordre de grandeur du conducteur, diminue très vite lorsque la largeur du conducteur augmente.

Les conducteurs électriques sont aussi utilisés pour créer des bulles par nucléation [28] ou pour les faire disparaître par collapse [29]. Dans cette application statique des conducteurs, on cherche à obtenir le maximum de champ magnétique pour le minimum de courant, ce qui revient à s'intéresser au maximum du rayon de la bulle. Sur la figure 6 , on a aussi représenté $R_{\mathrm{F}}$ qui est le rayon d'équilibre d'une bulle au bord du conducteur quand celui-ci est alimenté, en fonction de la largeur du conducteur. Cette courbe indique l'efficacité du conducteur pour transformer un courant donné en champ magnétique vertical au niveau d'une bulle. On voit que ce maximum a lieu pour des conducteurs dont la largeur est de l'ordre de grandeur du diamètre du domaine magnétique.

3.5 INFLUENCE DE L'INTENSITÉ DE L'IMPULSION. On sait que l'intensité de l'impulsion est un paramètre qui peut être facilement choisi lors de la conception d'une puce mémoire. En premier lieu nous avons regardé l'incidence de l'intensité du courant sur le temps de transfert. Sur la figure 7 pour un conducteur de $20 \mu$ de large et pour une bulle partant d'une distance de $120 \mu$, on a représenté le temps de transfert $\tau$ en fonction de l'inverse du courant appliqué. On obtient sensiblement une droite, ce qui signifie que le transfert se fait à $I \times \tau=$ Cte. Plus on exigera des fonctionnements à fréquence élevée, plus l'intensité des impulsions devra augmenter.

Puisque à la fin du déplacement, la bulle se trouve en $\left(x_{\mathrm{F}}, R_{\mathrm{F}}\right)$ dans un puits de potentiel créé par le courant, l'intensité de celui-ci va avoir une répercussion sur ce point d'équilibre. Sur la figure 8 sont représentées les valeurs de $x_{\mathrm{F}}$ et $R_{\mathrm{F}}$ pour un conducteur de $20 \mu$ de large
MATERIAU (SmTb) $\mathrm{FeO3}$

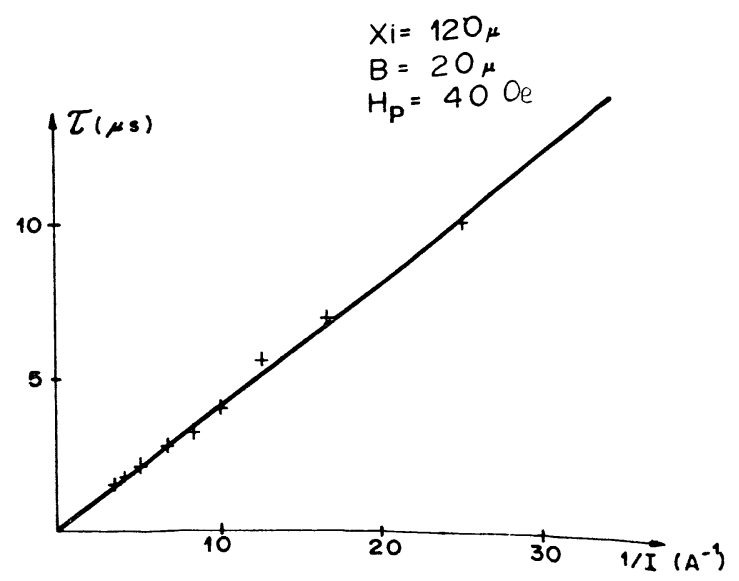

FIG. 7. - Action de l'amplitude de l'impulsion $I$ sur le temps de transfert $\tau$. ( + : calcul ; - : courbe $I \times \tau=$ Cte. $)$

MATERIAU (SmTb) $\mathrm{FeO}_{3}$

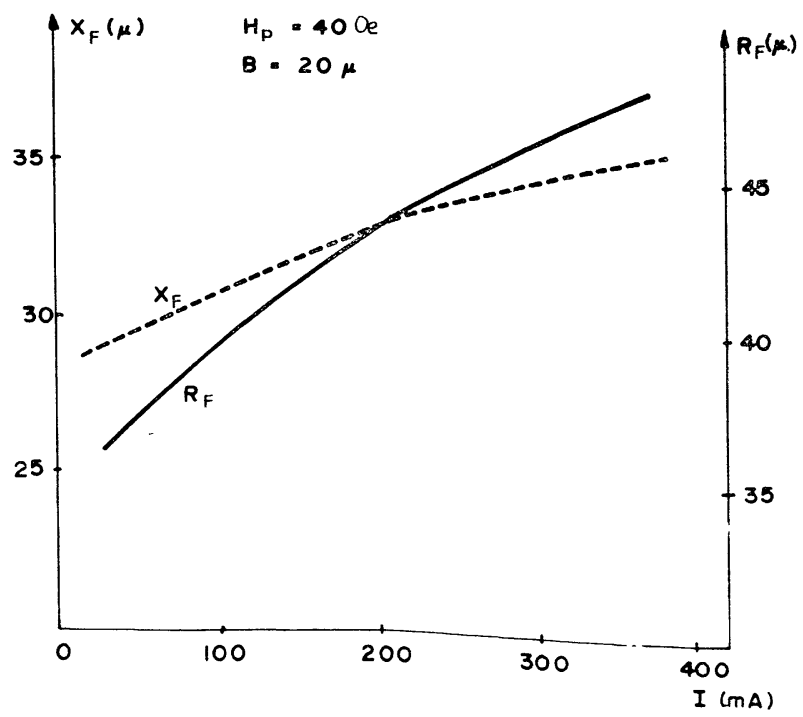

FIG. 8. - Influence de $I$ sur les coordonnées d'équilibre final $\left(x_{\mathrm{F}}, R_{\mathrm{F}}\right)$.

en fonction de l'intensité du courant appliqué pour un champ de polarisation de $40 \mathrm{Oe}$. Comme on pouvait s'y attendre, la valeur du rayon d'équilibre augmente régulièrement avec le courant, passant de $36 \mu$ à $47 \mu$, quand $I$ passe de $50 \mathrm{~mA}$ à $350 \mathrm{~mA}$. Parallèlement à cette variation du rayon d'équilibre, l'intégration des lignes de champ sur le volume de la bulle change. Ceci se répercute sur le point d'équilibre qui passe de $28 \mu$ à $36 \mu$.

Cette variation du point d'équilibre avec le courant dans le conducteur a des conséquences très importantes sur l'utilisation des conducteurs électriques. En se reportant à la figure 4 , on vérifie que la localisation des points $\mathrm{C}$ et $\mathrm{B}$ change avec le courant dans les conducteurs, et surtout que les points $\mathrm{C}$ et $\mathrm{B}$ ne seront jamais symétriques par rapport au conducteur. 
Si B s'éloigne du conducteur quand $I$ augmente (Fig. 8), C s'en rapproche. Par exemple, pour un conducteur de $20 \mu$ de large $I=200 \mathrm{~mA}$ et $H_{\mathrm{p}}=40 \mathrm{Oe}$. le point $\mathrm{B}$ est à $32 \mu$, alors que le point $\mathrm{C}$ est à $-27 \mu$. Ceci explique que la propagation des bulles soit impossible à réaliser uniquement par des conducteurs. En effet, supposons que par une impulsion négative on ait réussi à amener une bulle jusqu'au point B' B $^{\prime}$ pour qu'une impulsion positive continue à la propager, il faut qu'elle soit au moins au-delà du point $\mathrm{C}$. Il faut la faire passer du point $\mathrm{B}^{\prime}$ au point $\mathrm{C}$, soit à l'aide d'un plot de [6] permalloy soit à l'aide de gravures dans le motif de propagation lui-même. Les plots de permalloy sont placés sous les conducteurs de manière que le passage $\mathrm{B}^{\prime} \mathrm{C}$ soit assuré grâce à l'attraction du plot magnétique (Fig. 4).

Le principe des chemins de propagation gravés est que le domaine magnétique va se stabiliser là où l'épaisseur moyenne de la bulle sera la plus faible, c'est-à-dire où la surface gravée est maximum. Ce supplément de force permet de franchir la distance entre les points $\mathbf{B}^{\prime}$ et $\mathrm{C}$.

3.6 INFLUENCE DU CHAMP DE POLARISATION. - Le champ de polarisation agit sur le rayon des bulles, nous allons examiner de quelle façon ceci se répercute sur les temps de transfert et sur la position d'équilibre.

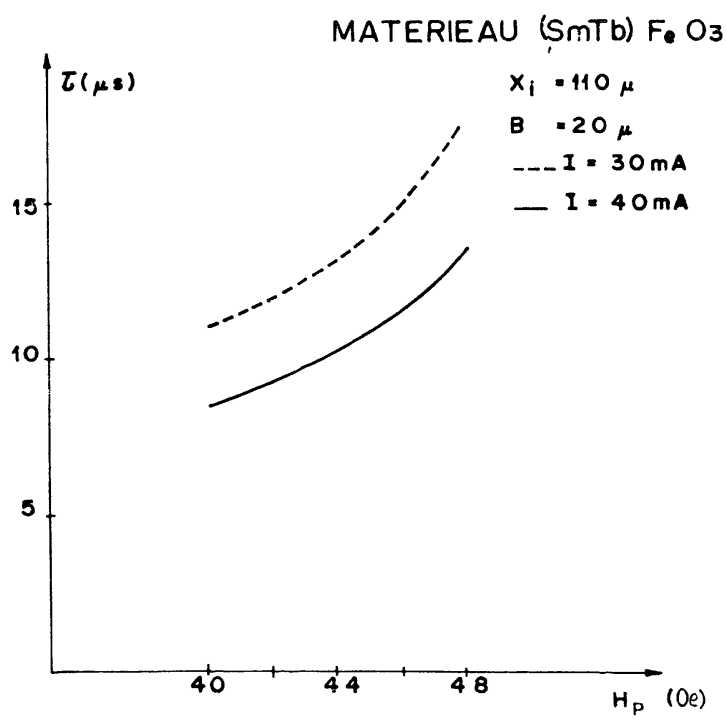

FIG. 9. - Influence du champ de polarisation $H_{\mathrm{p}}$ sur le temps de transfert $\tau$.

Sur la figure 9, on a représenté les temps de transfert pour un conducteur de $20 \mu$ de large alimenté par des courants de 30 et $40 \mathrm{~mA}$ et une bulle partant à une distance de $110 \mu$ de ce conducteur. Le temps de transfert augmente régulièrement avec le champ de polarisation, ce qui peut s'expliquer par le fait que le diamètre de la bulle diminue et par conséquent le gradient de champ entre la paroi avant et la paroi arrière de la bulle décroît, entraînant une diminution de la vitesse $\left(v=\frac{1}{2} \mu \times \Delta H\right)$.

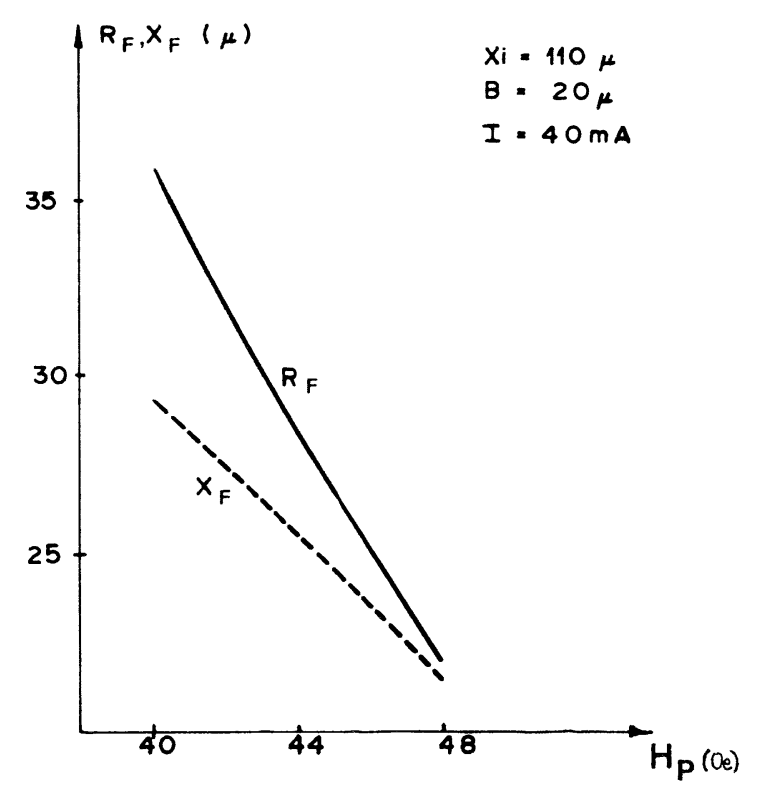

FIG. 10. - Influence du champ de polarisation sur les coordonnées d'équilibre final $\left(x_{F}, R_{F}\right)$.

Sur la figure 10 , on a tracé les coordonnées d'équilibre en fonction de $H_{\mathrm{p}}$, le rayon $R_{0}$ décroît régulièrement quand $H_{\mathrm{p}}$ augmente, ce qui entraîne parallèlement une diminution de $x_{0}$ : l'écartement du puits de potentiel dû à l'intégration sur le volume de la bulle diminue avec le rayon de celle-ci.

3.7 Diagramme synthétique. - Sur la figure 11, nous avons essayé de synthétiser les résultats précédents, pour en tirer quelques lois générales.

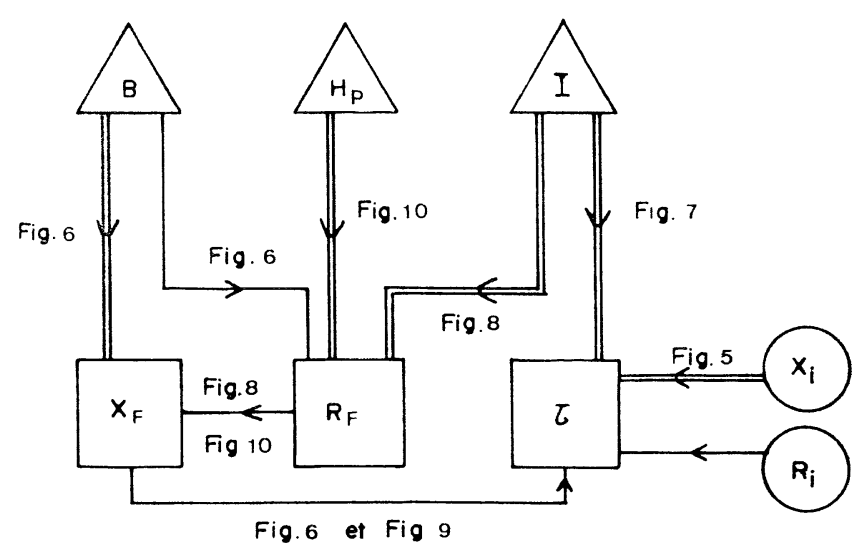

FIG. 11. - Diagramme synthétique.

Nous avons présenté les valeurs recherchées $x_{\mathrm{F}}, R_{\mathrm{F}}, \tau$ par des carrés, les paramètres de commande $B, H_{\mathrm{p}}$ et $I$ par des triangles et les valeurs initiales $x_{\mathrm{i}}$ et $R_{\mathrm{i}}$ par des ronds. Les actions directes sont représentées par des doubles flèches, les actions indirectes ou du second ordre par des flèches simples.

La position initiale $x_{\mathrm{i}}$ n'influence que le temps de transfert $\tau$, cette action est complexe quand $x_{\mathrm{i}}$ est au 
voisinage du conducteur, mais obéit à des lois simples si $x_{\mathrm{i}} \gg x_{\mathrm{F}}$ (Fig. 5). La largeur du conducteur $B$ influence directement $x_{\mathrm{F}}$ et de manière indirecte $R_{\mathrm{F}}$ et $\tau$ (Fig. 6). Le champ de polarisation et l'intensité de l'impulsion agissent sur le rayon d'équilibre (Fig. 8 et 10), ce qui se répercute sur la position d'équilibre. Une liaison simple existe entre le temps de transfert et le courant de commande (proportionnalité inverse) (Fig. 7). Par contre, la liaison entre le champ de polarisation et le temps de transfert se fait par l'intermédiaire du rayon.

4. Conclusion. - On s'est efforcé de traiter de manière aussi exacte que possible le problème du déplacement de domaines magnétiques cylindriques dans des gradients de champ. A partir d'un formalisme rigoureux basé sur l'utilisation d'une fonction de Lagrange et d'une fonction de dissipation de Rayleigh, nous avons établi le système différentiel du deuxième ordre qui décrit l'évolution du domaine en fonction du temps. On a montré qu'en raison de la valeur des coefficients qui y figurent, ce système peut se ramener à un système de premier ordre et on l'a appliqué au cas où le gradient de champ est créé par un conducteur électrique.

Le système correspondant de deux équations différentielles non linéaires couplées, ne pouvant se résoudre complètement de manière analytique, a été traité au moyen de calculs numériques.

L'exploitation systématique de ce programme nous a permis de mettre en évidence un certain nombre de phénomènes. En premier lieu, la courbe donnant la valeur du champ intégré sur le volume de la bulle en un point (le rayon d'équilibre en ce point étant atteint) n'est pas symétrique, ce qui interdit la propagation par courants bipolaires uniquement. Sur la façon d'utiliser un conducteur, il a été prouvé que la manière la plus efficace consistait à fair s passer une bulle d'un bord à l'autre du conducteur plutôt que de l'attirer de l'extérieur, ceci avec l'inconvénient que la distance parcourue est limitée à la largeur du conducteur. Pour celle-ci on a montré que l'optimum se situait entre une et deux fois le diamètre des bulles. On a vu que le champ de polarisation agissait de manière directe sur le rayon d'équilibre et de manière indirecte sur la position d'équilibre et le temps de transfert. Ceci signifie que les marges de fonctionnement d'un dispositif avec motifs de permalloy et conducteurs, dans le plan des phases (phase de l'impulsion, durée de l'impulsion) dépendent du point de fonctionnement choisi dans le plan des champs magnétiques (champ de polarisation, champ tournant).

Enfin, le courant de commande influe directement sur le temps de transfert, mais il a aussi une action du second ordre sur le rayon et la position d'équilibre.

Remerciements. - Les auteurs tiennent à remercier MM. Randet et Coeure pour leurs conseils, et MM. Mauduit, Perrin, Renard, Sauron pour leur contribution expérimentale.

\section{Bibliographie}

[1] Bonyhard, P. I., Geusic, J. E., Bobeck, A. H., Chen, Y. S., Michaelis, P. C., Smith, J. L., I. E. E. E. Trans. Magnetics MAG 9 (1973) 433-436.

[2] Copeland, J. A., J. Appl. Phys. 43 (1972) 1905-1908.

[3] George, P. K., Archer, J. L., J. Appl. Phys. 44 (1973) 444448.

[4] George, P. K., Archer, J. L., A. I. P. Conf. Proceedings $\mathrm{n}^{\circ} 10$ (1973) 222-226.

[5] George, P. K., Archer, J. L., I. E. E. E. Trans. Magnetics MAG 9 (1973) 303.

[6] Copeland, J. A., I. E. E. E. Trans. Magnetics MAG 8 (1972) 241-243.

[7] Goldstein, R. M., Shou,, M., Copeland, J. A., J. Appl. Phys. 44 (1973) 5090-5095.

[8] Yoshimi, K., Fujiwara, S., Yamauchi, F., Furuyoa, T., I. E. E.E. Trans. Magnetics MAG 8 (1972) 669-672.

[9] Chen, Y. S., Nelson, T. J., J. Appl. Phys. 44 (1973) 33063309.

[10] Gilbert, T. L., Phys. Rev. Abstr. 200 (1955) 1243.

[11] Schlomann, E., J. Appl. Phys. 43 (1972) 3834-3842.

[12] Henry, G. R., J. Appl. Phys. 42 (1971) 3150-3153.

[13] Rossol, F. C., J. Appl. Phys. 40 (1969) 1082-1083.

[14] Vella-Coleiro, G. P., Smith, D. H., van Uitert, L. G., J. Appl. Phys. 43 (1972) 2428-2430.
[15] Argyle, B. E., Malozemoff, A. P., A. I. P. Conf. Proceeding $\mathrm{n}^{\circ} 10$ (1973) 344-348.

[16] Woolhouse, G. R., Chaudhari, P., A. I. P. Conf. Proceeding, $\mathrm{n}^{\circ} 18$ (1974).

[17] Sertchik, J. A., Doyle, W. D., Goldberg, K. G., J. Appl. Phys. 42 (1971) 1272.

[18] Shumate, P. W., J. Appl. Phys. 42 (1971) 5770-5772.

[19] BoвecK, A. H., I. E. E. E. Trans. Magnetics MAG 6 (1970) 445.

[20] Rossol, F. C., Thiele, A. A., J. Appl. Phys. 41 (1970) 1163.

[21] Vella-Coleiro, G. P., Tabor, W. J., Appl. Phys. Lett. 21 (1972) 7-8.

[22] Vella-Coleiro, G. P., Hagedorn, F. B., Chen, Y. S., Blank, S. L., Appl. Phys. Lett. 22 (1973) 324-325.

[23] Vella-Coleiro, G. P., A. I. P. Conf. Proceedings $\mathrm{n}^{0} 18$ (1974).

[24] Rijnierse, P. J., de Leeuw, F. H., A. I. P. Conf. Proceeding $\mathrm{n}^{\circ} 18$ (1974).

[25] Hagedorn, F. B., A. I. P. Conf. Proceedings no 18 (1974).

[26] Rossol, F. C., I. E. E. E. Trans. Magn. MAG 7 (1971) 142144.

[27] Bonnes, G., Jouve, H., Mauduit, D., Revue Phys. Appl. 9 (1974) 501-502.

[28] Nelson, T. J., Chen, Y. S., Geusic, J. E., I. E. E. E. Trans. Magnetics MAG 9 (1973) 289-293. 